\title{
EFEITO DA DISTRIBUIÇÃO ESPACIAL DE INTENSIDADE DO LASER NOS PADRÕES DE SPECKLE DE SUPERFÍCIES METÁLICAS*
}

\author{
Marcella Mateus de Mello ${ }^{1}$ \\ Marcello Henrique Marques Passos ${ }^{2}$ \\ Marcelle Rodrigues Lemos ${ }^{3}$ \\ Sheila Rodrigues de Almeida ${ }^{4}$ \\ Wagner Franklin Bhaltazar ${ }^{5}$ \\ José Augusto Oliveira Huguenin ${ }^{6}$ \\ Ladário da Silva ${ }^{6}$
}

\section{Resumo}

O presente trabalho objetiva analisar a influência da distribuição de intensidade espacial do laser na geração de padrões de speckle em superfícies metálicas. Os padrões de speckle estudados são obtidos quando o laser atinge uma superfície metálica. A análise da imagem de padrões de speckle pode ser relacionada com a rugosidade de superfícies metálicas iluminadas com o laser. Com esse estudo pretendemos melhorar a sensibilidade das medidas de rugosidade de superfícies metálicas a partir das imagens de padrões de speckle.

Palavras-chave: Rugosidade; Padrão de speckle; Modo de Laguerre-Gauss; Modo de Bessel; Laser.

\section{LASER SPATIAL DISTRIBUTION INTENSITY EFFECT ON SPECKLE PATTERN OF METALLIC SURFACES}

\section{Abstract}

The present study aims to evaluate the influence of the laser intensity distribution in the generated speckle pattern. The studied speckle patterns are obtained when a laser beam shines a metallic surface. The fractal analysis of the speckle pattern images relates them with the metallic surface roughness. With this study we intend to improve the sensibility of the roughness measures on metallic surfaces.

Keywords: Roughness; Speckle Patterns; Laguerre-Gauss mode; Bessel mode; Laser.

1 Engenheira de Produção, Mestranda em Engenharia Metalúrgica, Programa de Pós-Graduação em Engenharia Metalúrgica (PPGEM), Escola de Engenharia Industrial Metalúrgica de Volta Redonda (EEIMVR), Universidade Federal Fluminense (UFF), Volta Redonda, RJ - Brasil.

2 Físico, Mestrando em Física, Pós-graduação em Física (PG-FIS), Instituto de Ciências Exatas (ICEX), UFF, Volta Redonda, RJ - Brasil.

3 Graduanda em Física, Coordenação de Física, ICEx, UFF, Volta Redonda, RJ - Brasil.

4 Acadêmica do Técnico em Automação Industrial, Instituto Federal de Ciência e Tecnologia do Estado do Rio de Janeiro (IFRJ), Volta Redonda, RJ - Brasil.

5 Físico, Doutor em Física, Professor, IFRJ, Volta Redonda, RJ - Brasil.

6 Físico, Doutor em Física, Professor, Departamento de Física, Instituto de Ciências Exatas (ICEx), UFF, Volta Redonda, RJ - Brasil. 


\section{INTRODUÇÃO}

A rugosidade é definida por um conjunto de desvios microgeométricos, caracterizado pelas pequenas saliências presentes em determinada superfície, ou seja, para avaliar a rugosidade é necessário ser feita uma análise da camada superficial do material. A correta avaliação da rugosidade é muito importante para o controle de qualidade em um processo de fabricação [1].

O fato de conseguir uma alta precisão nas medidas e não haver a necessidade de contato com as superfícies a serem analisadas são fatores pelos quais as técnicas ópticas têm sido bastante empregadas para caracterizar superfícies rugosas. $\mathrm{O}$ estudo de procedimentos que podem contribuir com o aumento da sensibilidade e precisão das medidas de rugosidades são importantes, haja vista essa medida ainda ser usada com um parâmetro de controle em diversos processos de controle de qualidade. O feixe laser habitualmente possui uma distribuição espacial de intensidade Gaussiana, identificada como modo Gaussiano. Ao se incidir um feixe laser em uma superfície, ocorre um espalhamento do feixe de luz sobre a superfícies devido a sua rugosidade. Essa luz espalhada possui uma distribuição de intensidade granular que, pode ser observada em ambos as direções especular e normal à incidência do feixe na superfície. Esta distribuição intensa é nomeada de padrão de speckle ou padrão speckle [2], e ela contém informações sobre a rugosidade na superfície do material. O padrão de speckle gerado por dispersão de luz coerente de uma superfície metálica rugosa tem sido explorado por diferentes abordagens, em nosso grupo de pesquisa, para se determinar a rugosidade da superfícies metálicas [3-7], que envolvem o cálculo de grandezas como por exemplo o expoente de Hurst $[3,6]$, a razão entre as regiões claras e escuras de uma imagem binarizada [4], a dimensão fractal do padrão speckle [5] e a limiarização entrópica usando a estatística de Tsallis em imagens de padrões de speckle [7]. Neste trabalho alteramos a distribuição espacial de intensidade do laser, passando-a para o modo de Laguerre-Gauss [8] e Bessel [9], e avaliamos seu efeito no padrão de speckle. Esse efeito pode contribuir para a melhorar a sensibilidade das técnicas anteriormente desenvolvidas.

\section{MATERIAIS E MÉTODOS}

Durante a realização das análises foram utilizadas amostras de alumínio polidas manualmente. A técnica de polimento manual utilizada baseou-se em polir a superfície das amostras com lixas de granulometria cada vez maior, em uma única direção até não haver mais os traços da lixa anterior. As lixas utilizadas possuem granulometria 80, 150, 320, 400 e $600 \mathrm{MESH}$.

Foi realizado um experimento descrito na Figura 1 para obtenção de imagens dos padrões speckle gerados pelas superfícies das amostras mostradas acima. De acordo com a Figura 1, um feixe laser (532 nm, $1 \mathrm{~mW})$ emite um feixe de luz o qual incide sobre um modulador espacial de fase - SPM (do inglês Spatial Phase Modulator). Em seguida passa pela Iris 11 e I2, de forma a marcar o caminho. A lente convergente $\mathrm{L} 1 \mathrm{com}$ distância focal de $100 \mathrm{~mm}$ controla o diâmetro do feixe de forma a iluminarmos uma determinada área da amostra. O espelho M1 direciona o feixe para incidir na amostra com um ângulo de $45^{\circ}$. A amostra é posta sobre um porta-amostra. A luz espalhada na direção normal à superfície da amostra é 
capturada pela câmera monocromática CCD que registra o padrão de speckle digital gerado por cada amostra.

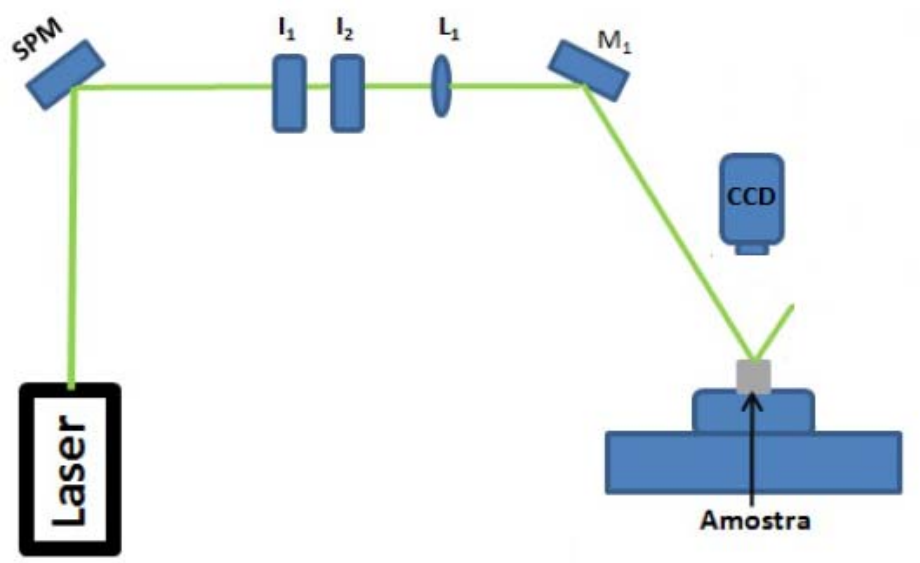

Figura 1. Esquema experimental da análise de padrões de speckle gerados pelo espalhamento do laser na superfície metálica.

O SPM é capaz de produzir modos transversos diferentes, como os conhecidos modos de Laguerre-Gauss [8], que possuem singularidade da fase e são conhecidos por vórtices ópticos, nos quais seu vetor de Pointing espirala em torno da origem do plano de propagação, assim como os modos de Bessel [9], nos quais o a distribuição transversa do campo elétrico é descrito por uma função de Bessel. A distribuição de intensidade do feixe no modo de Bessel se assemelha a uma série de anéis concêntricos.

A ideia é verificar se os padrões de speckle gerados por diferentes modos transversos produzem variações na distribuição de intensidade, capazes de alterar o contraste das imagens dos padrões de speckle das superfícies metálicas iluminadas, o que pode ser utilizado para aprimorar o cálculo da rugosidade via padrão de speckle.

O microscópio confocal (LEICA DCM3D) foi utilizado para avaliar a topografia da amostra. Se a amostra está um pouco inclinada, este fato pode alterar os resultados relacionados à medida de rugosidade usando o padrão speckle. Essa inclinação pode alterar o sinal capturado no plano normal. Com o microscópio é possível detectar este problema e se possível resolvê-lo, por exemplo, polindo novamente a amostra até a mesma obter uma superfície o mais plana possível, para não alterar os resultados na medida de rugosidade através do padrão speckle. Abaixo segue a figura 2 do microscópio confocal com uma das amostras posicionada para a análise de sua topografia. 

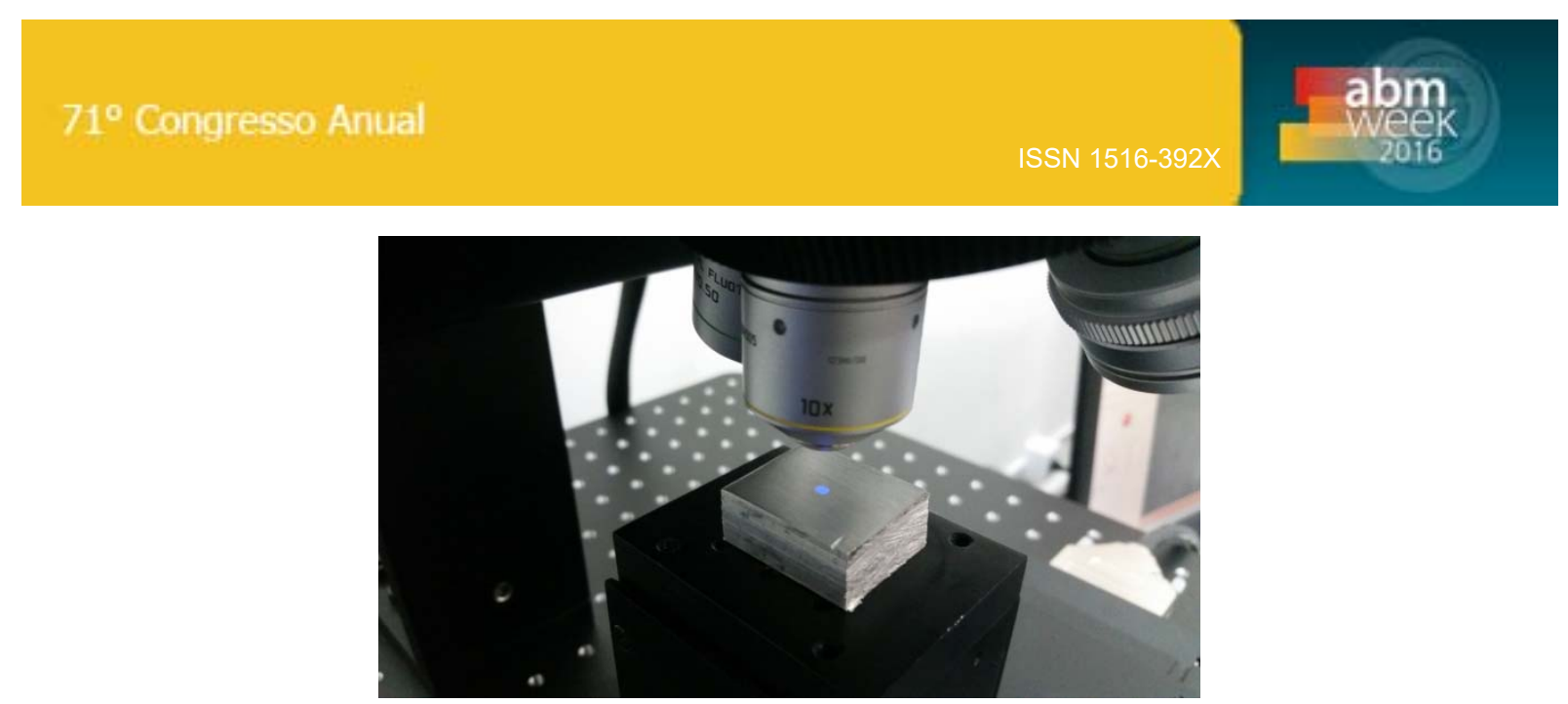

Figura 2. Análise da topografia da amostra no microscópio confocal.

\section{RESULTADOS E DISCUSSÃO}

As superfícies polidas foram analisadas no microscópio confocal afim de corrigirmos sua planicidade. A Figura 3 exibe uma superfície analisada.

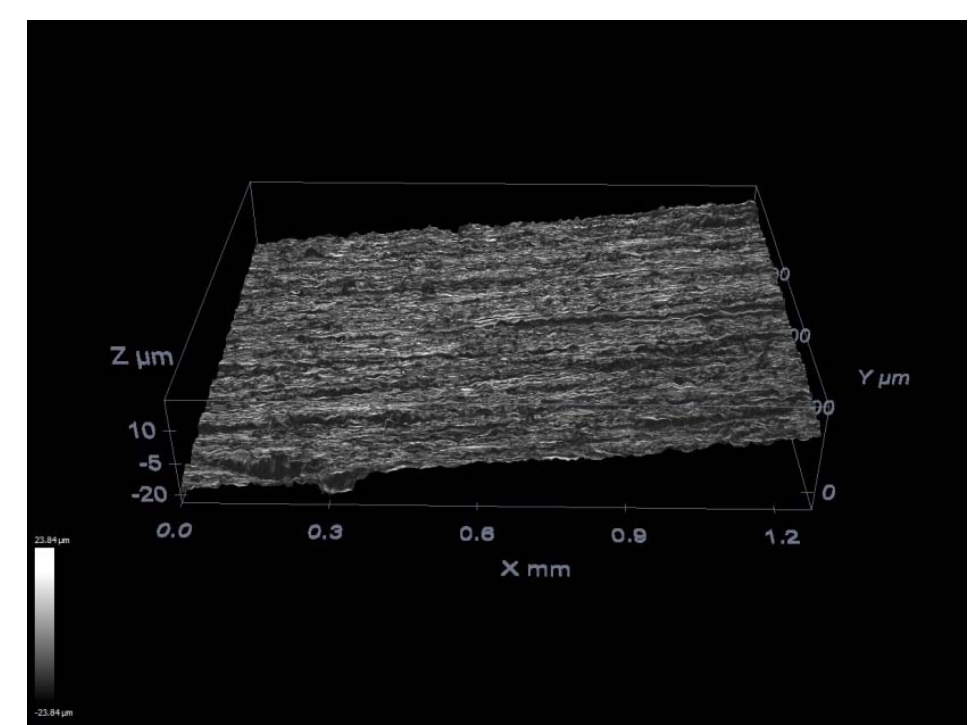

Figura 3. Levantamento topográfico de uma superfície metálica polida até a lixa 400

Após essa etapa as superfícies foram iluminadas com a laser, usando o modo Gaussiano, o modo Laguerre-Gauss e o modo de Bessel no processo descrito anteriormente pelo esquema da Figura 1. Para isto todos os demais parâmetros foram mantidos constantes, como a potência do laser, geometria da montagem, condições de iluminação e etc. Após as superfícies metálicas das amostras serem polidas com lixas de granulações diferentes até a última lixa de interesse: $400 \mathrm{MESH}$ ou $600 \mathrm{MESH}$, elas foram postas no porta-amostra mostrado na Figura 1. As imagens capturadas na direção normal dos padrões de speckle, com os diferentes modos (distribuições espaciais) são exibidas na Figura 3. 
a)

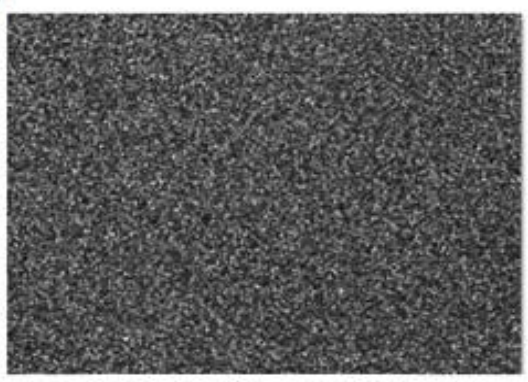

Gaussiano 400

b)

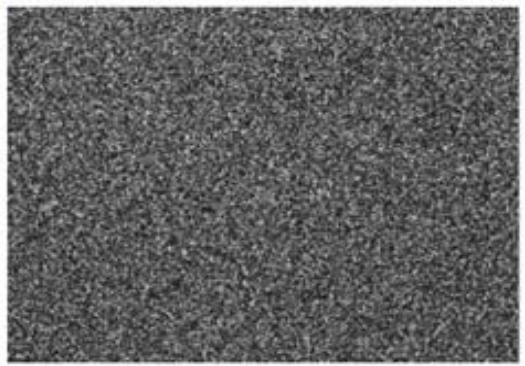

Laguerre +1 400

c)

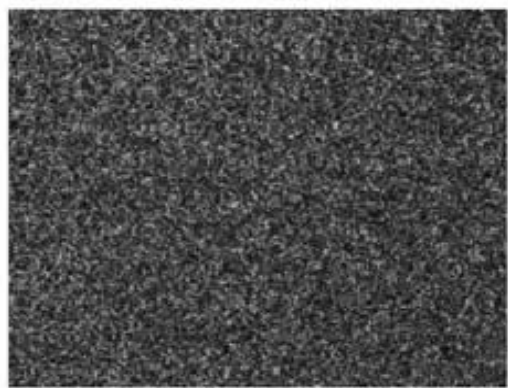

Bessel 0400

d)

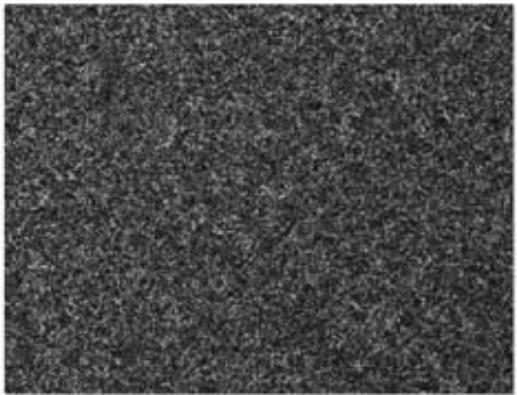

Bessel 3400

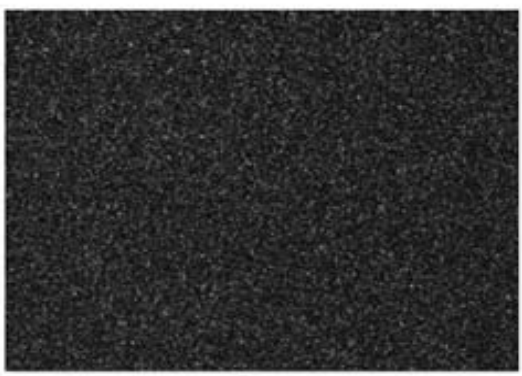

Gaussiano 600

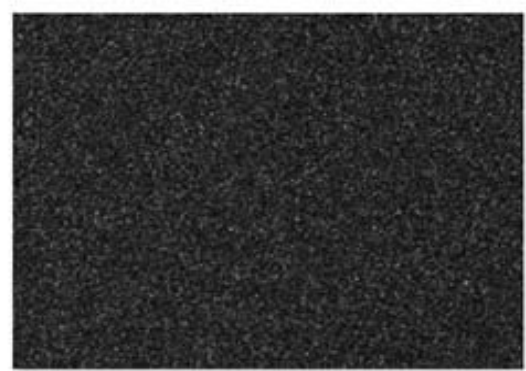

Laguerre +1 600

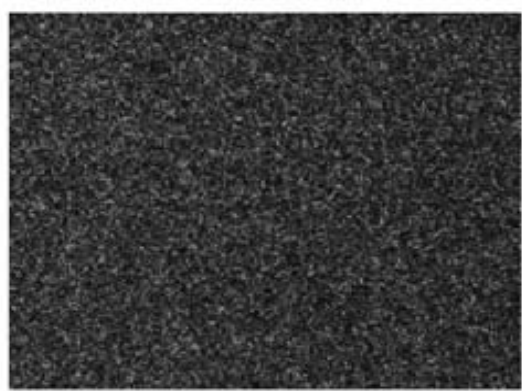

Bessel 0600

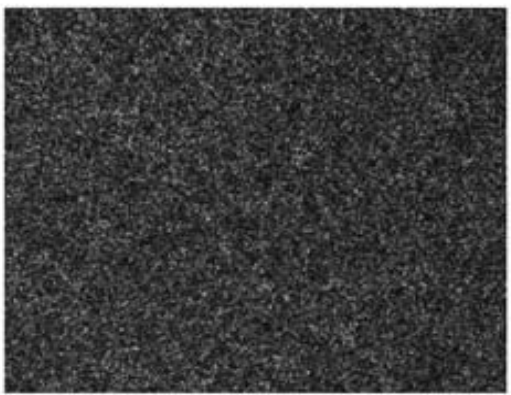

Bessel 3600

Figura 3. Imagem digital do padrão speckle das amostras de Al. (a) Modo Gaussiano das amostras polidas com $400 \mathrm{MESH}$ e $600 \mathrm{MESH}$. (b) Modo Laguerre-Gauss das amostras polidas com 400 MESH e $600 \mathrm{MESH}$. (c) Modo Bessel, primeira ordem, das amostras polidas com $400 \mathrm{MESH}$ e 600 MESH. (d) Modo Bessel, segunda ordem, das amostras polidas com $400 \mathrm{MESH}$ e $600 \mathrm{MESH}$. 
Nessas imagens, as condições de iluminação, potência do laser, posição da amostra, etc., ficaram fixas, mudando-se apenas as distribuições de intensidade do laser. Comparando as imagens da mesma superfície iluminadas com diferentes modos, percebemos que há uma diferença no contraste e iluminação das mesmas. A amostra polida até a lixa de $400 \mathrm{MESH}$, que se encontra na primeira coluna da Figura 3, apresenta uma variação evidente de sua iluminação e contraste, quando iluminada por modos diferentes. O mesmo pode ser percebido na segunda coluna, onde se encontra a amostra polida até a lixa $600 \mathrm{MESH}$.

Observando agora a variação de intensidade e contraste entre as superfícies polidas com 400 e 600 MESH em uma mesma linha, portanto iluminadas com o mesmo modo, percebemos que os modos Gaussiano e Laguerre-Gauss de primeira ordem apresentam maior variação de intensidade e contraste que o de Bessel de ordem zero e terceira ordem. Neste caso a diferença é atribuída às diferentes rugosidades das mesmas. Assim, concluímos que iluminar amostras rugosas como modos transversos diferentes gera diferenças no padrão de speckle, o que pode influenciar no cálculo indireto de rugosidade. Estamos implementando o estudo para mais amostras com acabamentos com lixas diferentes das apresentadas, a fim de gerarmos uma análise diferencial mais significativa desse efeito. De qualquer forma esse procedimento pode ser empregado em diferentes superfícies. Para cada uma delas será necessário testar o modo que permite maior contraste. Espera-se assim que os resultados obtidos em [3-7] possam apresentar maior sensibilidade em relação aos valores dos diferentes parâmetros empregados para acessar a rugosidade de superfícies metálicas.

\section{CONCLUSÃO}

Neste trabalho verificamos que diferentes modos distribuição espacial de intensidades do feixe, como o modo Gaussiano, o modo de Laguerre-Gauss e o modo de Bessel causam uma mudança no padrão de speckle gerado. Isso permite vislumbrar uma nova maneira de se capturar o padrão de speckle a fim de processálo para o cálculo da rugosidade, de modo a que o parâmetro empregado apresente maior sensibilidade em relação ao valor da rugosidade da superfície metálica analisada.

\section{Agradecimentos}

Os autores agradecem às agências brasileiras: Conselho Nacional de Desenvolvimento Científico e Tecnológico (CNPq) - Projeto 314685/2009-0, Fundação Carlos Chagas Filho de Amparo à Pesquisa do Estado do Rio de Janeiro (FAPERJ) - Projetos E26/110.321/2012 e E26/110.655/2013, à Pró-Reitoria de Pesquisa, Pós-Graduação e Inovação (PROPPI) da Universidade Federal Fluminense (UFF) - Projeto 23069.004676/2014-97 pelo apoio financeiro, e à Coordenação de Aperfeiçoamento de Pessoal de Nível Superior (CAPES), CNPq e FAPERJ pelas bolsas de alguns dos alunos. 


\section{REFERÊNCIAS}

1 ABNT-NBR ISO 4287. Especificações geométricas do produto (GPS) - Rugosidade: Método do perfil - Termos, definições e parâmetros de rugosidade. 2002.

2 Goodman JW. Some fundamental properties of speckle. J. Opt. Soc. Am.1976; 66: 1145.

3 Sampaio AL; Lobão DC; Nunes LCS, dos Santos PAM, Silva L., Huguenin, JAO. Hurst exponent determination for digital speckle patterns in roughness control of metallic surfaces. Optics and Lasers in Engineering. 2011;49:32-35.

4 Meireles JB, da Silva L, Caetano DP, Huguenin JAO. Effect of metallic surface roughness on the speckle pattern formation at diffraction plane. Optics and Lasers in Engineering. 2012;50:1731-1734.

5 Corrêa RD, Meireles JB, Huguenin JAO, D.P. Caetano, L. da Silva. Fractal structure of digital speckle patterns produced by rough surfaces. Physica A. 2013;392:869-874.

6 Soares HC, da Silva L, Lobão DC, Caetano DP, Huguenin JAO. Hurst exponent analysis of moving metallic surfaces. Physica A. 2013;392:5307-5312.

7 Soares HC, Meireles JB, Castro Junior AO, Huguenin JAO, Schmidt AGM. Tsallis's Threshold Analysis of Digital Speckle Patterns Generated by Rough Surfaces. Physica A. 2015;432:1-8.

8 Padgett M, Allen L. Light with a twist in its tail, Contemporary Physics. 2000;41:275285.

9 Duocastella M, Arnold CB. Structured light for processing. Laser Photonics Rev. 2012;6(5): 607-621. 